\title{
HACIA UN PERFIL DEL INTELECTUAL DIGITAL: LA EXPRESIÓN RECUPERADA DE GOMBROWICZ
}

\author{
TOWARDS A PROFILE OF THE DIGITAL INTELLECTUAL: THE \\ GOMBROWICZ'S EXPRESSION RECOVERED
}

Enrique Ferrari Nieto

Universidad de Extremadura. España/Spain

eferrari@unex.es

\author{
Recibido/Received: 20/11/2011 \\ Modificado/Modified: 05/10/2012 \\ Aceptado/Accepted: 11/10/2012
}

\begin{abstract}
RESUMEN
Coinciden en nuestro tiempo la impresión generalizada del fin del intelectual clásico y el nacimiento de nuevos foros de opinión menos restrictivos debido a las posibilidades de edición y difusión de Internet. Ante la reacción suspicaz que arremete contra estas nuevas voces, cabe plantear -para dar con una imagen más equilibrada- las posibilidades del intelectual digital para protegerse, con su papel redefinido, de los peligros que han amenazado siempre su autonomía. En esta tarea, para acertar con el perfil del nuevo intelectual, mi propuesta es una relectura de la reflexión de Gombrowicz sobre los modos de la cultura y su exhibición.
\end{abstract}

\section{PALABRAS CLAVE}

Internet, autoría, software libre, cultura.

\section{SUMARIO}

1. Las fricciones entre intelectuales e Internet. 2. El otro enfoque para la cultura, de Gombrowicz. 3. Internet, tierra abierta a la inmadurez. 4. El lenguaje hipertextual, frente a los recelos con lo virtual. 5. El reverso de las críticas. Bibliografía.

\begin{abstract}
There is a general feeling of the end of the classical intellectual. At the same time there are new less restrictive opinion forums on the Internet. There is a suspicious reaction lambasting these new voices. On the opposite side, we can analyze how the digital intellectual can defend themselves against the dangers which threatened their autonomy. In this task, to draw the profile of the new intellectual, I propose a reading of Gombrowicz's reflection on the ways of culture and its display.
\end{abstract}

\section{KEYWORDS}

Internet, authorship, free software, culture.

\section{CONTENTS}

1. Frictions between intellectuals and Internet. 2. The other approach to culture, by Gombrowicz. 3 . Internet, open to immaturity. 4 . The hypertext language, against the fears with virtuality. 5 . The back of the accusations. References. 


\section{LAS FRICCIONES ENTRE INTELECTUALES E INTERNET}

En estos últimos años dos discusiones convergen en un mismo escenario: una, de mayor recorrido, sobre la desaparición de los grandes intelectuales, o del papel que asumieron en tiempos pasados (Jacoby, 2000); y la otra, más reciente, sobre la legitimidad y reputación de las nuevas voces que aparecen por primera vez con Internet, trastocados del todo los ámbitos de la edición y la comunicación. En ambos casos, la postura mayoritaria es, todavía, la nostálgica o la escéptica, más cómoda mirando hacia atrás que hacia delante, segura en sus dominios. Con argumentos a tener en cuenta, como la idoneidad de los formatos digitales para recoger y transmitir un texto de envergadura. Pero con una premisa en origen falsa, equivocada, de los riesgos digitales para el autor (digamos, profesional), que incapacita a sus partidarios, a menudo tan reacios y tan ciegos, para equilibrar la balanza con su reconocimiento a los méritos y capacidades de la edición en red, muchas veces la solución (o una mejora considerable) a quejas remotísimas.

Una premisa, como digo, falaz, porque confunde dos términos: el derecho de autor y el copyright. El derecho de autor es uno de los derechos fundamentales en la Declaración Universal de los Derechos Humanos. Reconoce que la obra es expresión del autor, de la persona que es el autor; que hay una identidad entre este y su creación. Con lo que lo protege, como un derecho moral, reconociéndole su autoría, como fuente de una propiedad intelectual. Pero la noción del derecho anglosajón de copyright, que a veces se confunde con el derecho del autor, solo comprende la parte patrimonial de esos derechos de autor: como tal, es solo un derecho de propiedad comerciable, limitado estrictamente a la obra, sin considerar lo que el autor ha volcado de sí mismo en su creación. No es, propiamente, un derecho del autor: no lo protege de nada, simplemente lo convierte en propietario, garantizándole un porcentaje de los ingresos de dicha obra. Y es aquí, solo en este ámbito, donde se plantean los cambios (o los riesgos, según la perspectiva) a los que obliga Internet con una reformulación de la propiedad intelectual que puede o debe adoptar algunas de las fórmulas de la denominada cultura del software libre. Porque nunca se ha cuestionado para Internet los derechos del autor -exactamente los mismos en uno u otro formato-, sino el copyright, lo referido a su dimensión patrimonial, en principio para productos informáticos, pero también para cualquier otro producto cultural, que tiene, como alternativas para la red, el copyleft (un modo de ejercer el derecho de autor que consiste en permitir la distribución libre de copias y versiones de un trabajo a cambio de que se preserven esos mismos derechos en las copias modificadas) y los wiki (sitios Web que los usuarios pueden editar con total libertad al instante, para hacer de la edición un trabajo colectivo). Con el reconocimiento moral, en ambos casos, a sus autores. Pero también con la propuesta -ineludible- de trastocar ese viejo concepto de autor, nacido con el libro de papel, que choca con la tecnología actual de la edición (Negroponte, 1995:79).

Porque esta noción de autoría ha tenido, de la mano de la responsabilidad individual, un desarrollo lento, tardío (arranca en el Renacimiento, pero hasta el XVIII no se consolida), con ataques, casi desde el principio, muy fuertes. Pero solo ahora se pone en duda, o entre paréntesis, para Internet, ante una noción más globalizadora de autoría colectiva, que recuerda a los textos abiertos y sin autor que proponía Condorcet (aunque luego reculó) para divulgar el conocimiento, que debían circular libremente entre los ciudadanos: un conocimiento a partir de los sentidos, decía el francés, que no puede pertenecer a nadie, como si fuera una propiedad privada, porque el conocimiento es propio de la naturaleza misma, no de la mente que lo percibe (Nunberg, 1998:26-30). Porque el 
aura del autor ha ido de la mano de las limitaciones para publicar, para divulgar las ideas a un público amplio. Pero una vez corregidas estas, una vez que se ha expandido el acceso a la distribución masiva por la red, como ha escrito Javier Echeverría, se modifica también nuestra comprensión de lo que significa ser autor y qué clase de autoridad se puede atribuir (Echeverría, 1999:184). Lo que condiciona, a su vez, la pervivencia de los considerados intelectuales, los que antes tenían a su disposición, en exclusiva, los medios. Porque ahora Internet, la blogosfera, en la que se superponen distintas voces, les quita el monopolio al resolver de golpe la escasez endémica que padecían los medios de expresión. Escribe Molinuevo: "Internet declara al intelectual clásico como especie amenazada, le cuestiona. Internet sería, sí, la imagen del nihilismo: el desierto (Internet) crece y los intelectuales se recluyen como islas o bien luchan por sobresalir en el piélago" (Molinuevo, 2006:132).

En esta etapa de transición, mientras perdura el libro pero Internet le va ganando terreno cada día, con pocas excepciones el intelectual clásico se ha mantenido, con el término de Negroponte, como un indigente digital, sin apenas contacto con las nuevas tecnologías, o con aproximaciones muy tímidas, tomadas solo como un complemento menor del libro o de la cátedra, sin luchar por conquistar los nuevos ámbitos de la comunicación y la edición. Probablemente solo por una cuestión de edad, por no haber nacido más tarde. Pero mientras, a la cabeza de la transición, voces hasta ahora no reputadas, por lo general mucho más jóvenes, y muchos más en número (todos somos intelectuales, decía Gramsci), unidas por la noción de cultura participativa o de saber colectivo o de inteligencia colectiva, o libre, organizados en red (Kerckhove, 1998:32), comienzan a ocupar el hueco reservado hasta hace muy poco a estos. Primero han construido su espacio, como programadores informáticos, como hackers (Castells, 2001:56-67), hasta dar con una plataforma única, de todos y para todos, y, luego, con esa misma actitud de colaboración, de libertad y de voluntad de ir mejorando el producto, se han volcado en los contenidos, que comparten y distribuyen libremente en blogs, foros, revistas digitales, archivos, etc. Algunos muy valiosos y otros menos. Como los libros o las revistas de papel. Aunque sin esa primera criba que suponen los costes de la edición y distribución clásicas. Pero la cuestión, aquí, es cómo, garantizados en Internet esos derechos del autor (incluso reforzados, al desligarlos del mero derecho a copia), se fortalecen también algunos de los supuestos derechos del intelectual, no recogidos en ningún documento, pero fácilmente identificables. Porque la diferencia entre un autor y un intelectual es la actividad pública del segundo. Todo intelectual es un autor, pero no todos los autores quieren para sí ese perfil del intelectual (ahora del intelectual específico, con el término de Foucault). Necesitan esa exposición, esa presencia suya fuera de su ámbito específico de conocimiento; y ahí, en ese mostrarse, están los riesgos, que hay que rebajar con unos derechos mínimos, uno básico para preservar su independencia, su autonomía: su protección ante el uso y manipulación del poder, político y económico (que se maneja tan mal con Internet).

En su diagnóstico del intelectual del siglo XX Sartre es demoledor: "Los intelectuales son débiles por naturaleza: no producen y para vivir solo cuentan con su salario, lo que les imposibilita defenderse tanto en la sociedad civil como en la sociedad política. Helos aquí, pues, ineficaces y volubles; a falta de un poder económico o social, se autoconsideran una elite llamada a juzgar acerca de todo, algo que no son" (Sartre, 1987:92). Como francotiradores, escribe Edward Said, impotentes ante el entramado de autoridades sociales (Said, 1996:17). Para Ortega había sido una cuestión de deserción, una acción (o una omisión) puntual en un momento complicado, solo eso, falta de compromiso o de saber 
estar a la altura, pero Sartre -el paradigma- lo lleva a su naturaleza, a su propia condición de intelectual, fallida, imposible, porque está obligado a atarse a aquello que debe criticar, en una relación demencial, en la que se imponen demasiados intereses a la crítica imparcial. No da una respuesta. No es fácil dar con ella. Pero una aproximación teórica al intelectual tomado no como sujeto (con sus funciones, naturaleza, etc.) sino como objeto, como el que también recibe una acción, puede, al menos, mostrar otra perspectiva para un primer balance. No como guía (sujeto), sino como potencial marioneta (objeto). Y desde ahí sabemos que solo el hecho de incrementar exponencialmente el número de individuos que divulgan y comparten información le complica mucho al poder sus mecanismos de control (Castells, 2001:70). Aunque las pegas estén también claras, con la baja calidad de muchas de las publicaciones (una cuestión menor) y (esto mucho más grave) el nivel altísimo de infosaturación. Con la cuestión de fondo que en El hombre sin atributos de Musil plantea el jefe de oficina de prensa a Tuzzi: “Digámoslo otra vez: ¿dónde está el límite entre lo que se toma en consideración y lo que se pasa por alto?" (Musil, 2010:217).

\section{EL OTRO ENFOQUE PARA LA CULTURA, DE GOMBROWICZ}

Postman, convertido ya en clásico, recupera del Fedro de Platón la medicina para el recuerdo, no la memoria, la sabiduría no verdadera, hecha solo de información (Postman, 1994:14). Y González Quirós el asno de Buridán, que muere de vacilar entre el agua y la avena (González Quirós, 1998:64). Žižek habla de anorexia informativa como reacción al exceso de información (Žižek, 2006:254). Y Maldonado de una opulencia informativa con los mismos efectos para el usuario que la anterior indigencia (Maldonado, 1998:99-100). En frente, como contrapeso, del lado de las capacidades de la edición en red, me voy a ceñir a un solo ejemplo, a una reivindicación con casi cien años de historia: A comienzos del XX, en los arrabales de la vanguardia, Witold Gombrowicz publica Ferdydurke como respuesta a la reacción que en determinados ámbitos culturales polacos causa un libro suyo anterior, Memorias del tiempo de la inmadurez. Lo tachan, en un ejercicio de perspicacia, de inmaduro, y Gombrowicz, con su segundo intento, ajusta cuentas con la cultura que se arrogan estos popes: tan hermética, tan inflamada, tan venerable, y (sin la contradicción que podría parecer evidente) también tantas veces solo una excusa. Le hace una crítica por dentro y por fuera: con el análisis del mecanismo interno de la cultura y con la reflexión de su uso público, de su exhibición que hace el intelectual, ambos conectados.

Con Ferdydurke, para su primera crítica, opone lo inmaduro a la Forma en un movimiento dialéctico, pero de una dialéctica insegura, dubitativa, que quiere a un tiempo lo perfecto y lo imperfecto, la cultura y la vida, sin ser capaz de resolver la antítesis. En palabras de su autor: "Añadir a todos los altares oficiales otro más sobre el que se irguiese el dios joven de lo inferior, de lo peor, de lo sin-importancia, en todo su poder vinculado con lo bajo" (Gombrowicz, 2006:66). En síntesis, cree que los hombres se ven obligados a ocultar su inmadurez; que solo exteriorizan lo que está maduro en ellos. Pero esa madurez que muestran es solo una ficción que no se corresponde con lo que ellos son: inmaduros por su propia naturaleza. Y también por la cultura: "La cultura -escribe- infantiliza al hombre porque ella tiende a desarrollarse mecánicamente y, por lo tanto, le supera y se aleja de él" (Gombrowicz, 2010:17). Porque el hombre persigue todo el tiempo la Forma, pero nunca la logra. Como en el Ferdydurke, en el que es el anhelo de madurez lo que arrastra al hombre hacia esa inmadurez artificial; y ese anhelo deforma el que lo lleva a 
una forma mala: porque se precipita en su búsqueda, incapaz de vivir sin ella, y se equivoca, igual que el hombre vergonzoso que, al encontrarse desnudo, no acierta a coger ropa de su talla. Lo que quiere el protagonista es encontrar la forma para la Inmadurez. Imposible, porque no puede manifestar directamente su propia realidad inmadura. Con lo que lo único que puede hacer es tomar distancia frente a la forma para, al menos, dice, volver menos cargante la cultura: esa máscara que la convierte en un juego mecánico. Como advierte el propio Gombrowicz: "Si no lográis juntar de algún modo más estrecho esos dos mundos, la cultura será siempre para vosotros instrumento de engaño" (Gombrowicz, 2010:17).

En la segunda de sus críticas, con Trans-Atlántico, que escribe mucho después, lleva el conflicto al entorno de los intelectuales: de un lado los que buscan amarrarse a las referencias y las citas de los prohombres del pasado, porque entienden la cultura como la acumulación de datos; y, del otro, los que rechazan tantos andamios para expresar sus ideas, que quieren más próximas: Una primera demostración de independencia, digamos, interna, para el trabajo propiamente intelectual, teórico, que Gombrowicz también alienta en el plano político como la bisectriz que separa al intelectual cercano al poder del intelectual que decide romper amarras: en ambos casos una posición difícil. Escribe: “¡Cuán extraño, extrañísimo, aquel Caso! Sabía que eran unos comemierdas y que me consideraban como a un comemierda y todo aquello no era sino mierda, mierda, y lo único que deseaba era romperles la Cabeza a aquellos comemierdas. Sin embargo, no se trataba de un cualquiera, sino del Ministro Plenipotenciario, el Señor Ministro, y su Consejero... A eso se debía mi Timidez, mi temor ante Personas tan importantes que me distinguían y honraban... Así que cuando en aquel Salón el Ministro y el Consejero me asaltaron con Homenajes mientras corrían detrás de mí, yo, sabedor de la Alta Misión, de la dignidad e importancia de aquellos comemierdas, no podía rechazar ni librarme de aquel Homenaje. ¡Vaya, había caído como una ciruela en medio de la mierda!” (Gombrowicz, 1986:25-26).

Desde aquí, con esta doble mirada, recuperado Gombrowicz en la era de Internet, su reflexión -compartida por otros, pero en su caso más visceral, más vivida- puede servir para buscarle esa otra perspectiva a la nueva figura de lo que he llamado el intelectual digital, en Internet. Porque junto a tantos ataques cabe también, desde el soporte conceptual que supone la noción de archivo que apunta Boris Groys, una legitimación de sus formas o al menos una valoración positiva de algunos de sus rasgos más destacados. La premisa es que con Internet y la tecnología digital, al multiplicarse casi ilimitadamente la capacidad de almacenaje de información, ya no se da, porque no se necesita, esa competición que ha existido hasta hace poco para imponer cada uno sus ideas. Antes, sin los mecanismos apropiados para acumular todas las propuestas, tenían que luchar entre ellas para imponerse en ese espacio mínimo que era la cultura o la alta cultura, para pervivir; y las opciones de cada una pasaban por alinearse con la cultura ya consagrada, como heredero legítimo. Pero hoy, con la cultura del pensamiento libre, con tantos teras para acumular datos, con los márgenes de lo tolerable mucho más amplios, la cosa ha cambiado, con la integración de otras tradiciones y otras propuestas. El libro de papel, que restringió tanto la autoría, está siendo sustituido por otros formatos informáticos, infinitamente más baratos, que hacen posible que cualquiera pueda divulgar sus ideas: que a cualquiera puedan leerlo en cualquier parte del mundo. Aunque a estos no se les reconozca mayoritariamente como intelectuales, aunque no gocen del mismo estatus, ni del mismo respeto. En principio porque no son ya minoría, y porque en este grupo tan heterogéneo los hay que no van más allá de copiar y pegar lo que han leído en otro sitio o 
porque los argumentos que plantean son irrelevantes o incoherentes, meras ocurrencias, sin una base sólida. Pero también -y esto no ha sido estudiado- porque la percepción inicial que se tuvo de Internet como otro mundo, como algo virtual, sin consecuencias, como si tuviera una entidad ontológica menor, condicionó una rebaja en la consideración de dichos autores y publicaciones, como una segunda división respecto al libro de papel.

\section{INTERNET, TIERRA ABIERTA A LA INMADUREZ}

La clave que arranca esta reflexión, con la traslación inevitable, es de Roberto Bolaño: "Gombrowicz supo ver en Argentina esa cualidad del exilio y para el exilio: una tierra en donde la Forma se deshace constantemente, tierra no historiada, es decir tierra abierta a esa libertad y a la inmadurez" (Bolaño, 2008:54). Un punto de inflexión. Un nuevo punto de partida sin deudas con lo anterior. Gombrowicz había llegado a Buenos Aires en 1939, en el viaje inaugural de un transatlántico, el Chrobry, para una estancia de solo unos días, pero la segunda Guerra Mundial y la invasión de Polonia lo retuvieron en Argentina 24 años, al principio en la miseria, y sin escribir, alejado de los círculos literarios que Victoria Ocampo gestionaba. Con Ferdydurke, que escribe cuando todavía vivía en Polonia, busca en los tumbos de su protagonista una actitud para él, que no ve clara, en un equilibrio difícil entre el hechizo que le causa la juventud, la inmadurez, la joven -encantadorainferioridad y la reputación de los grandes valores, la orgullosa, pero trágica y nada atractiva, madura superioridad. De un lado la tierra virgen, sin deudas, la creación propia que no debe nada a nadie (o no más que lo inevitable), más atada a la vida que a las formas, y del otro la cultura como la suma de valores adquiridos en la que la forma se impone, con los moldes ya prefijados para cualquier contenido. Otra vez la elección desazonadora de los románticos. De un lado los valores, la forma, y del otro la inmadurez, la juventud: La aspiración sensata, un hueco en la pléyade, o la insensata atracción de la inmadurez eternamente rejuvenecedora; también la perfección y la imperfección, los valores y los subvalores: "El libro -escribe él mismo en su diario- es la imagen de alguien que, enamorado de su inmadurez, pugna por la madurez" (Gombrowicz, 2006:58). Quince años después, ya en Argentina, retomada la escritura, con Trans-Atlántico fija su posición en esa tierra de nadie. Conecta el comienzo de la novela con su biografía, con su llegada a América como un escritor reconocido, pero la historia se bifurca con la noticia del comienzo de la guerra: el Gombrowicz real se diluye en el anonimato y la pobreza en Buenos Aires; el Gombrowicz ficcional, en cambio, se presenta en la Embajada para, de algún modo, ofrecerse como intelectual, un incordio al principio para el embajador, que lo humilla, cansado de tantos ofrecimientos ("No soy una vaca para que me ordeñen"), hasta que le descubre posibles usos, exhibiéndolo para hacer publicidad patria. Hasta que, en la escena decisiva, a Gombrowicz lo enfrentan a un escritor argentino (probablemente Eduardo Mallea), que aplasta cada uno de sus argumentos con citas y referencias para despojarlo de toda originalidad, con las influencias que encuentra en su vasta (cargante) cultura. Entonces huye del Embajador y su séquito. Es el punto de inflexión. Porque se ve empujado, casi sin darse cuenta, al lugar de la barbarie, para quedarse con lo inferior, con lo inmaduro, con lo no desarrollado.

Lo que conecta con la observación de Bolaño, y también con Borges, al que lo vincula Piglia como escritores de literaturas secundarias y marginales, en la periferia de la cultura europea, que les permite, dice, la posibilidad de un manejo propio, irreverente, de las 
grandes tradiciones, un uso propio, muy particular, de la herencia cultural (Piglia, 1997:1315). La tierra no historiada de Bolaño es la que no le reclama deudas con los antepasados, liberado, de algún modo, de una cultura excesiva, apabullante, la cultura que supera al hombre y lo ahoga, lo que para Ortega era la cultura hieratizada, solidificada, mostrenca, adiposa, no germinal. Es la tierra donde la forma no se ha impuesto definitivamente: un hábitat (aunque la metáfora es peligrosa) que tiene, tantos años después, otros dominios más sugerentes en Internet. Lo que, para Groys, en Sobre lo nuevo, es el espacio profano, lo otro que queda fuera de la cultura: "El ámbito que integra todas las cosas que no están incluidas en los archivos" (Groys, 2007:76). Lo que no tiene valor, o no tiene interés, lo irrelevante. Pero -por esto mismo, por ser la reserva de valores culturales potencialmente nuevos- también lo que hace posible la innovación, al trastocar la jerarquía de valores que es la cultura (que en seguida lo asimila y neutraliza como amenaza). Ahora, con una cultura técnicamente archivada, con otros mecanismos u otras motivaciones; porque el autor, dice Groys, ya no busca que sus ideas sean de obligado cumplimiento en el futuro, que ejerzan una influencia decisiva, como sí querían antes los que se proponían innovar: imponer sus ideas al mayor número de individuos, para que se mantuvieran en el tiempo. Al autor contemporáneo le basta con que se le reconozcan como una nueva aportación. Que el reconocimiento de que su propuesta es novedosa, distinta a las demás, le permita tener su espacio. Porque, escribe Groys, "hoy, la memoria histórica de un determinado autor no se debe tanto a la victoria absoluta de sus ideas; se debe, en mayor medida, al sistema universal de archivos en forma de -señala entonces- bibliotecas y museos" (Groys, 2007:53-54): los contenedores culturales tradicionales, con muchos siglos detrás, a los que hay que sumar ahora -porque el libro lo publicó en 1992, hace veinte años- Internet: un paso más, decisivo, en el sistema de almacenaje y gestión de la información.

\section{EL LENGUAJE HIPERTEXTUAL, FRENTE A LOS RECELOS CON LO VIRTUAL}

Solo queda superar algunas suspicacias en torno a la virtualidad real -con el término de Castells- de Internet. Pero no va a ser fácil. Porque no lo es seguirle el paso a la tecnología informática, una vorágine que lo alcanza todo, en cada campo convertida en su columna vertebral, desde hace veinte o treinta años a una velocidad demencial con la que crea y deshecha las nuevas aplicaciones casi a un tiempo, con pocos meses de diferencia: lo que tardan en quedar obsoletas. Unos plazos a los que no estábamos acostumbrados. Tampoco para la reflexión, a la que le cuesta caminar tan rápido: a un tiempo no perder de vista sus movimientos y procurar un análisis certero, de verdad reflexivo, a una distancia prudencial para no perder su autonomía, y no convertirse tampoco en mera ocurrencia. Pero la tecnología digital no permite otro ritmo que no sea el suyo. Y, con estas, es fácil precipitarse, analizar un fenómeno aislándolo en exceso, sin tener una visión completa del hecho tecnológico, o, al contrario, generalizando para cuestiones muy diferentes, sin precisar los rasgos distintivos de cada una de sus materializaciones. Lo fue al menos al principio, cuando no había de dónde agarrarse y hubo que construir el entramado teórico desde cero. Para una definición de Internet, la proa de las tecnologías de la información y la comunicación, algunos de los estudios iniciales lo vincularon erróneamente con lo virtual (sin más especificaciones) y, por tanto, con la simulación, con la apariencia, con lo no real; o bien para reafirmar la tesis de Baudrillard de los simulacros y lo hiperreal para 
un tiempo en que la verdad ha quedado, también, obsoleta, o bien, confiados todavía en cierta fortaleza ontológica de la realidad, para denunciar el fraude de la confusión de lo real y lo no real en Internet.

Obviaron lo propiamente técnico, su sistema hipertextual, como un conjunto descentralizado de redes de comunicación interconectadas, y lo emparentaron con la realidad virtual, otro mecanismo informático, desde fuera más popular, o más impactante $\mathrm{y}$, por esto, mejor atendido por la ciencia ficción, la gran divulgadora de la tecnología, con películas como Desafío total, de Paul Verhoeven, en 1990, o Matrix, de Wachowski, en 1999. Con el término, con ese 'virtual' más restrictivo, los informáticos al principio diferenciaban la máquina física de la máquina con la que los usuarios creían que se comunicaban; pero de siempre, en su definición, virtual es lo que tiene existencia aparente y no real, lo que produce un efecto, como si fuera real, sin ser real, lo que -dejados a un lado los excesos de Baudrillard- ha llevado a algunos a enfocar el tema hacia los riesgos del engaño, con la aparición de imágenes ficcionales que no remarcan su carácter ficcional, que pueden confundirse con otras reales, exactamente iguales: fakes, con el término en inglés: falsificaciones, montajes, con los que se construye una información falsa o un texto que puede entenderse, equivocadamente, como información. Los teóricos que emparentaron Internet con lo virtual, desde sus comienzos, lo obligaron a asumir esas mismas pegas de lo virtual, como si fuera una enfermedad congénita. Como si Internet fuera otro mundo, con esa imagen tan intuitiva que nace de la dicotomía entre lo analógico -el mundo en el que vivimos- y lo digital -otro distinto, intangible, al que se accede solo con los terminales, con el interfaz del ordenador o la tableta o el teléfono móvil. Uno y otro separados, con legislaciones diferentes. Uno a la fuerza serio, con consecuencias reales para cada acción, y el otro, en principio, más permisivo, sin una relación directa con nuestra vida, fuera de nuestra órbita de responsabilidades, con una tecla capaz de deshacer lo hecho si no nos gusta el resultado.

Con lo que un intelectual digital, el intelectual propio de Internet, tendría también que hacer frente a los reparos de su apellido, ganarse una legitimidad extra: además de la que debe ganarse cualquier intelectual, por sí mismo, con sus ideas, un plus suplementario para vencer las reticencias a su medio que, en principio, ni siquiera es tomado por muchos como real: aquí, sin disquisiciones ontológicas, sinónimo de grave. En cualquier caso, todavía hoy, como un medio mucho menos prestigioso que el libro de papel, como si todos los sitios Web fueran iguales y se merecieran la misma consideración, la misma suspicacia.

Manuel Castells ha ceñido muy bien el sentido de los dos adjetivos, virtual y real, sumados, ahora que van de la mano. Escribe en La galaxia Internet: "Es virtual porque [nuestra cultura] está construida principalmente mediante procesos virtuales de comunicación de base electrónica. Es real (y no imaginaria) porque es nuestra realidad fundamental, la base material con la que vivimos nuestra existencia, construimos nuestros sistemas de representación, hacemos nuestro trabajo, nos relacionamos con los demás, obtenemos información, formamos nuestra opinión, actuamos en política y alimentamos nuestros sueños. Esta virtualidad es nuestra realidad" (Castells, 2001:230). Pero aún así a muchos teóricos todavía se les aparece el internauta, bajo su marco teórico, como el hombre desencarnado de McLuhan: con poco peso y muy veloz, sin una identidad privada, atrapado, como avisó el propio McLuhan, en una realidad quimérica (McLuhan y Powers, 1990:103). Como el hombre sin atributos, ubicado en la posibilidad, sin conceder más importancia a lo que es que a lo que no es. Sin atributos, porque disfrutar de atributos presupone una cierta deleitación en la realidad: "Tales hombres de la posibilidad -escribió 
Musil- viven, como se suele decir, en una tesitura más sutil, etérea, ilusoria, fantasmagórica, subjuntiva" (Musil, 2010:18).

\section{EL REVERSO DE LAS CRÍTICAS}

No han sido capaces de verle el reverso a este intelectual internauta. De verle, junto a sus posibles defectos como guía, también su resistencia a comportarse como una marioneta: un elemento también de su compromiso. De ver en este intelectual colectivo la mejor defensa frente al poder, por emplear el mismo mecanismo que hace funcionar a Internet: la conexión de nodos, la estructura reticular que sobrevive sin problemas al fallo o la corrupción de alguno de estos nudos. De ver, en último término, con el fin de esa figura que añoran, también el fin de muchas de sus limitaciones: las obligaciones anexas, inevitables, en el papel del intelectual clásico, el exceso de sobo colectivo, que decía Ortega con ese regusto aristocrático suyo (Ortega, 2005:IV, 102), pero también otras posibilidades menos amables, como la manipulación, la extorsión o la difamación. Que ahora ese intelectual digital rechaza, inmerso en una colectividad, diluidos en buena medida los nombres propios, al no reclamar para sí los derechos arrogados por los prohombres que lo precedieron, ese protagonismo social que para este no es más que un apéndice menor, prescindible, de su trabajo: un incordio que desaparece a la fuerza cuando los intelectuales (o los sustitutos de los intelectuales pasados) son tantos, y con jornadas solo parciales. Los intelectuales amateur (Said, 1996:114): Los culpables últimos, para los que entienden como una lacra la infosaturación de Internet. Pero también el torrente que rompe los viejos cauces de la cultura para abrirla y empaparla más de lo cotidiano de la vida, con temas nuevos o retomados: Los que han acudido al reclamo de inmadurez de Gombrowicz.

\section{BIBLIOGRAFÍA}

BOLAÑO, R. (2008), Entre paréntesis. Barcelona: Anagrama.

CASTELLS, M. (2001), La galaxia Internet. Barcelona: Areté.

ECHEVERRÍA, J. (1999), Los Señores del aire: Telépolis y el Tercer Entorno. Barcelona: Destino.

GOMBROWICZ, W. (1986), Transatlántico. Barcelona: Anagrama.

GOMBROWICZ, W. (2006), Diario argentino. Buenos Aires. Adriana Hidalgo Editora.

GOMBROWICZ, W. (2010), Ferdydurke. Barcelona: Seix Barral.

GONZÁLEZ QUIRÓS, J. L. (1998), El porvenir de la razón en la era digital. Madrid: Síntesis.

GROYS, B. (2007), Sobre lo nuevo. Ensayo de una economía cultural. Valencia: Pre-textos.

JACOBY, R. (2000), The last intellectuals. New York: Basic Books.

KERCKHOVE, D. (1998), "Inteligencia conectada y mente colectiva". En Revista de Occidente 206.

MALDONADO, T. (1998), Crítica de la razón informática. Buenos Aires: Paidós.

MCLUHAN, M., y POWERS, B. R. (1990), La aldea global. Barcelona: Gedisa.

MOLINUEVO, J. L. (2006), La vida en tiempo real. La crisis de las utopías digitales. Madrid: Biblioteca Nueva.

MUSIL, R. (2010), El hombre sin atributos. Barcelona: Seix Barral.

NEGROPONTE, N. (1995), El mundo digital. Barcelona: Ediciones B.

NUNBERG, G. (comp.) (1998), El futuro del libro. ¿Esto matará eso?. Barcelona: Paidós.

ORTEGA Y GASSET, J. (2005), “El poder social”. En Obras completas (tomo IV). Madrid: Taurus. 
PIGLIA, R. (1997), “¿Existe la novela argentina? Borges y Gombrowicz”, en Espacios de crítica y producción, 6, Buenos Aires.

POSTMAN, N. (1994), Tecnópolis. Barcelona: Círculo de Lectores.

SAID, E. W. (1996), Representaciones del intelectual. Barcelona: Paidós.

SARTRE, J. P. (1987), Escritos políticos. El intelectual y la revolución. Madrid: Alianza.

ŽIŽEK, S. (2006), Lacrimae Rerum. Madrid: Debate.

\section{Breve currículo:}

\section{Enrique Ferrari Nieto}

Doctor en Filosofía, licenciado en Filología Hispánica por la Universidad de Valladolid, estudios que ha completado en Temple University (Filadelfia) y UNAM (México DF). Es investigador posdoctoral de la Universidad de Extremadura. Ha sido profesor asistente de Ética aplicada a la Sociología en la UCU de Montevideo, investigador visitante en el Instituto de Filosofía (CSIC) y profesor visitante en las Universidades de Ljubljana y Turín. Ha escrito el libro Diccionario del pensamiento estético de Ortega $y$ Gasset (Mira Editores, 2010). 